\title{
Special Focus on Family Caregiving
}

Vida Vaitonis

$\mathrm{F}$ amily caregivers are no longer an invisible army, isolated and alone, tucked away in their homes silently caring for loved ones. They're organizing themselves, speaking up, recognizing their efforts are "work" and setting their own standards. We, the professionals in the health system, need to support them and acknowledge the critical role they play.

It is with thoughtful optimism that I invite you to read this issue's articles on family caregiving, as the papers provide insight into emerging policy changes and leading practices that hold promise in making a difference to caregivers' realities.

Themes throughout the papers cover the challenges caregivers face in a fragmented and unreliable system. Predominant are access issues, affordability, a lack of knowledge or awareness of educational and available resources and a paucity of muchneeded human and material resources and tools.

Unpaid caregivers in our healthcare system deserve our recognition and support. Our challenge is to create a new beginning for the support, recognition and inclusion of the caregiver in the newly adopted focus on "person-centred experience." The stability of our system depends on it.

So what's the "state of the affairs" for family caregivers in Canada? In reviewing the following papers submitted on the subject, several haunting questions were raised for me.

\section{What's the Problem?}

Caregivers are tired and literally sick to death of the overwhelming role required of them (Family Caregiving Alliance 2006). The burden on family caregivers has historically been mostly women who may have their own health issues in addition to family obligations. They rarely mind hard work and competing priorities. The "burden" is estimated at roughly between $\$ 30$ and $\$ 40 \mathrm{~B}$ worth of "free" labour (Hollander et al. 2009). But what are the true "costs" to each of these women's lives?
The demographics and trends suggest that if we don't figure out how to recognize their implicit value and ease the burden, we may lose them. Where will that leave those of us who require or will require their care?

\section{What's the Gap?}

As is true for any of us doing any job, volunteer or otherwise, it is a basic human need to feel competent, confident and valued.

A starting point is to acknowledge that the role caregivers' play is not only invaluable to the cared-for-person but also crucial to the quality and financial sustainability of the health system. The national strategy developed by the Canadian Caregiver Coalition (2008) needs sound traction, starting with a cultural shift in thinking akin to the mindset of redesigning care around the patient experience.

From the title of the article, "Caregiving Is Not a Disease," one might suppose that someone suggested it was. Notwithstanding, evidence exists where caregiving can result in disease and even death, owing to the burden and profound stress one experiences. Authors, Holyoke and Giosa, document the journey Saint Elizabeth Health Care undertook directly with family caregivers in every phase of the work to change its culture to "move from a reactive mode to a proactive model of support for caregivers across the healthcare system." These authors introduced the concept of caregiving as a "career," something to be considered with its pros and cons.

\section{What Do Caregivers Need?}

Just ask them. They undoubtedly know what they need and it's not what you might think. Like all of us, being in charge of the process and decisions that impact us, makes a significant difference to designing effective solutions. 
In its investigation of the merits of the Caregiver Framework in the article on high-needs children, Peckham et al. focus on their family caregivers and describe the "range of negative consequences" on caregivers from earlier discharge policies and non-institutional care options. Under the direction of the caregivers themselves, service plans were developed to take advantage of a range of new offerings, dedicated system coordinators, modest discretionary funds and single-care plans. Their outcomes demonstrate enhanced family caregivers' confidence and ongoing resilience in the caregiving role.

New language such as "cared-for-persons" and "supported self-management" as well as the concept of a "caregiving career" signal shifts in the cultural attitudes towards legitimatizing and acknowledging the value of caregivers in the health system.

\section{What's Fair and Reasonable?}

We seem paralyzed to figure out the fair and reasonable balance between public responsibilities versus private obligation as we debate whether the state has a role in what has always been a private family obligation. In the first article, Williams and colleagues raise the significant question of what should we expect from caregivers and what should they expect from us in return? "Expectations are, at best murky, arbitrary and conditional" with no "service guarantees."

Not surprisingly, they re-confirm the link between determinants of health and socioeconomic factors, albeit wondering if "poverty leads to disability," or "disability leads to poverty" with an unresponsive fragmented "non-system," making it all worse for those least able to cope with the added burdens of chronic illness.

\section{How Should We Support Family Caregivers?}

In a similar initiative to support caregivers caring for older persons, Warrick et al. document the value of the Caregiver Framework for Seniors Project (CFSP). Undertaken by a group of Toronto community agencies, individualized care plans were co-designed by the care coordinators and family caregivers using interventions outside of the normal basket of services including modest discretionary funds caregivers could use as they saw fit.

Above all else the approach recognized family caregivers as "clients" in their own right and recast the relationship between the formal and informal caregiver. The family member became an "active" and educated participant in "formal care decisionmaking" leading to a sense of mastery and control over one's situation and overall better support.

\section{So What Are the Risks in Being a Caregiver at Home?}

Rounding out the theme on caregivers is a paper by Doran et al., centring on safety at home. They highlight a "new" area for investigation for policy and practice changes in light of recent evidence of adverse events in the home care sector for various factors that include "risky decisions" made by clients and family caregivers.

It is no news to family caregivers that delivering care inside one's home is a risky business. The metrics used to measure the risk may need to include the risk to the caregivers themselves.

The articles and initiatives validated the vital role of unpaid family caregivers. Appeals are put forward to "think beyond a short-term fix, to forward plan" and pay attention to lessons learned: engage much earlier ... avoid the crisis. HQ

\section{About the Guest Editor}

Vida Vaitonis MEd, BScN, is president of V2 Associates Inc. consulting firm, past founding CEO of Mississauga Halton Community Care Access Centre, former director of Home and Community Care Branch of MOHLTC, former executive director of Ontario Long Term Care Association, Ontario Home Health Care Providers Association and Ontario Home Care Programs Association. Vida has served on numerous committees with mandates to better serve Ontario's most vulnerable citizens including Children with Special Needs; frail Seniors; persons with disabilities; Elders experiencing Abuse and Alzheimer's; and was instrumental in launching Ontario's first-ever End-of-Life Strategy in 2004.

\section{References}

Canadian Caregiver Coalition. 2008. A Framework for a Canadian Caregiver Strategy. Canadian Caregiver Coalition. Retrieved September 4, 2014. <http://www.cccccan.ca/content.php?doc=23>.

Doran, D., R. Blais, G.R. Baker, M.B. Harrison, A. Lang, M. Macdonald, J. McShane and T. Killackey. 2014. "The Safety at Home Study: An Evidence Base for Policy and Practice Change." Healthcare Quarterly 17(3): 42-47 doi: 10.12927/hcq.2014.24019.

Family Caregiver Alliance. 2006. Caregiver Health. San Francisco, CA: Family Caregiver Alliance. Retrieved September 4, 2014. <https:// www.caregiver.org/caregiver-health>.

Giosa, J. and P. Holyoke 2014. "Caregiving Is Not a Disease: Moving from Reactive to Proactive Supports for Family Caregivers across the Healthcare System." Healthcare Quarterly 17(3): 36-41 doi: 10.12927/ hcq. 2014.24018.

Hollander, M.J., L. Guiping and N.L. Chappell. 2009. "Who Cares and How Much? The Imputed Economic Contribution to the Canadian Healthcare System of Middle-Aged and Older Unpaid Caregivers Providing Care to the Elderly." Healthcare Quarterly 12(2): 42-49. doi:10.12927/hcq.2009.20660

Peckham, A., K. Spalding, J. Watkins, C. Bruce-Barrett, M. Grasic and A. P. Williams. 2014. "Caring for Caregivers of High-Needs Children." Healthcare Quarterly 17(3): 30-35 doi: 10.12927/hcq.2014.24017.

Warrick, N., A. Peckham, J. Watkins, M. Padjen and A.P. Williams. 2014. "Caring for Caregivers of High-Needs Older Persons." Healthcare Quarterly 17(3): 24-29 doi: 10.12927/hcq.2014.24016.

Williams, A.P., A. Peckham, J.Watkins, N. Warrick, T. Tam, D. Rudoler and K. Spalding. 2014. "Caring for Caregivers: Facing up to Tough Challenges." Healthcare Quarterly 17(3): 20-23 doi: 10.12927/ hcq.2014.24015. 